\title{
Application of human factors engineering (HFE) to the design of a naloxone auto-injector for the treatment of opioid emergencies
}

\author{
Robert B. Raffa ${ }^{1}$ - Robert Taylor Jr. ${ }^{2}$ (D) Joseph V. Pergolizzi Jr. ${ }^{2,3} \cdot$ Srinivas Nalamachu ${ }^{4}$. \\ Eric S. Edwards ${ }^{5}$ Evan T. Edwards ${ }^{5}$
}

Published online: 25 August 2016

(C) Controlled Release Society 2016

\begin{abstract}
The increased use of opioids for chronic treatment of pain and the resulting epidemic of opioid overdoses have created a major public health challenge. Parenteral naloxone has been used since the 1970's to treat opioid overdose. Recently, a novel naloxone auto-injector device (EVZIO, kaleo, Inc., Richmond, VA) was approved by the Food and Drug Administration. In this article, we review the Human Factors Engineering (HFE) process used in the development and testing of this novel naloxone auto-injector currently used in nonmedical settings for the emergency treatment of known or suspected opioid overdose. HFE methods were employed throughout the product development process for the naloxone auto-injector including formative and summative studies in order to optimize the auto-injector's user interface, mitigate use-related hazards and increase reliability during an opioid emergency use scenario. HFE was also used to optimize the product's design and user interface in order to reduce or prevent user confusion and misuse. The naloxone auto-injector went through a rigorous HFE process that included perceptual, cognitive, and physical action analysis; formative usability evaluations; use error analysis and summative design validation studies. Applying HFE resulted in the development of a
\end{abstract}

Evan T. Edwards

Evan.Edwards@kaleopharma.com

1 Temple University School of Pharmacy, Philadelphia, PA, USA

2 NEMA Research, Bonita Springs, FL, USA

3 Department of Medicine, Johns Hopkins University School of Medicine, Baltimore, MD, USA

4 International Clinical Research Institute, Overland Park, KS, USA

5 Kaleo, Inc., Richmond, VA, USA product that is safe, fast, easy and predictably reliable to deliver a potentially life-saving dose of naloxone during an opioid overdose emergency. The naloxone auto-injector may be considered as a universal precaution option for at-risk patients prescribed opioids or those who are at increased risk for an opioid overdose emergency.

Keywords Naloxone · Auto-injector · Opioid overdose · Human factors engineering · Opioid-induced respiratory depression

\section{Introduction}

Increasing awareness of the mechanisms of pain in humans and an ethical obligation to provide greater pain control [1] have driven increased prescribing of opioids. From 1991 to 2013, the number of prescriptions for opioids have increased from approximately 76 million to 207 million in the United States [2]. Drug poisoning deaths attributable to opioid analgesics quadrupled from about 4000 in 1999 to over 16,000 in 2010 [3]. In 2014, there were 18,893 fatalities from prescription opioid overdoses. The vast majority $(83 \%)$ of fatalities are unintentional $[4,5]$. Moreover, opioid deaths now exceed the number of deaths for both suicide and motor vehicle accidents combined [6]. In a study of unintentional overdoses in West Virginia from 1999 to 2004, opioid analgesics were taken by $93 \%$ of decedents, but only $44 \%$ had prescriptions for those drugs [7]. The majority of opioid-associated unintentional deaths $(60 \%)$ occur in patients prescribed opioids according to current prescribing guidelines [6], are prescribed opioids by a single practitioner [8], and do not have a substance-abuse disorder diagnosis [9]. The majority of opioid overdose deaths occur at home and are witnessed, including deaths associated with prescribed opioids [10]. In 2010, a total 
of 135,971 visits to US emergency departments occurred for opioid overdose, but this represents only a fraction of the total number of opioid overdoses as many individuals do not present to the emergency department or die before reaching emergency care [11]. This epidemic of opioid overdose also impacts children in the US as, on average, approximately 3300 children $\leq 5$ years old are admitted to emergency departments each year due to opioid exposure and poisonings [12].

During opioid-induced respiratory depression (OIRD) resulting from an opioid overdose, brain cells are starved of oxygen and start to die within about four minutes, which in many situations is much sooner than the average emergency response time (approximately $9 \mathrm{~min}$ ) [13-15]. Thus, there is a clear need for an opioid overdose antidote that is designed and intended to specifically be used by laypersons, with or without training, outside of a healthcare setting.

Naloxone hydrochloride, an opioid antagonist, was initially approved by the US Food and Drug Administration (FDA) in 1971 as an emergency medication indicated for the diagnosis or treatment of suspected opioid overdose [16]. Naloxone is generally administered intravenously, intramuscular or subcutaneously due to its low bioavailability via oral routes and high 1st pass metabolism [17]. Naloxone also rapidly crosses the blood brain barrier due to its high lipophilicity [17]. Once in the central and peripheral nervous system, naloxone exerts its function by altering the binding affinity and ligand-receptor binding kinetics of many opioids as well as receptor messaging [17]. Clinical use of naloxone grew rapidly due to a number of key properties, which include strong affinity for the $\mu$ receptor, rapid reversal of central and peripheral effects of opioids, minimal side effects at doses 100-fold above therapeutic levels and its inability to produce respiratory depression [17]. Naloxone hydrochloride became the standard of care for treating life-threatening OIRD [18]. Due to differences in opioids, repeated naloxone doses may need to be administered to reverse OIRD or central nervous system (CNS) depression emergencies. There are no contraindications to naloxone hydrochloride other than hypersensitivity to naloxone or to any of the ingredients in the formulation. Injectable naloxone may be administered to pediatrics, including neonates, and during pregnancy when the potential benefits outweigh the risks. Administration of naloxone to reverse life-threatening opioid-related respiratory depression may cause a sudden and complete reversal of opioid effects and precipitate withdrawal symptoms including irritability, confusion, or nervousness.

Previously, there were no FDA approved stand-alone naloxone prescription products on the market specifically labeled for use in non-medical settings such as the home and by nonmedical personnel, including caregivers or family members of patients. Until recently, only three realistic scenarios existed in which an individual in the community setting might receive naloxone during a suspected opioid emergency: (1) the naloxone is administered by an emergency responder, (2) the patient is taken to the hospital where naloxone is administered in the hospital emergency department, or (3) naloxone is administered using a naloxone kit from a harm reduction program [19]. Administering emergency naloxone from a naloxone kit requires the user to be familiar with the products and proper administration technique, including being able to draw the correct dose from a vial into a syringe or assembling an improvised multi-component injectable naloxone system adapted for intranasal (IN) delivery, and then properly administering the dose(s) to the patient [20]. Since emergency naloxone is by definition administered in high-stress emergency situations, untrained bystanders may feel overwhelmed by these requirements. Furthermore, the unprotected needles in some of these systems could unnecessarily expose the individual who is administering the naloxone to the patient to blood-borne pathogens.

Effective use of naloxone for opioid overdose populations has been demonstrated in community-based distribution programs. In a study of 2500 subjects from 2010 to 2013 , naloxone distribution resulted in 702 opioid reversals and the naloxone refills were most likely obtained by those who had witnessed an overdose and those who used heroin or methamphetamine [21]. An overdose education and naloxone distribution (OEND) program implemented in 19 New England communities reported training of 2912 potential bystanders and a total of 327 rescues [22]. Other community-based overdose prevention programs (OPPs), such as Project Lazarus, have been similarly effective in preventing and reversing potentially life-threatening opioid overdoses [23, 24]. Thus, structured programs offering access and training for prompt and appropriate administration of naloxone are a life-saving, effective strategy to help reduce opioid-related deaths.

Most of the aforementioned community-based programs utilize kits consisting of injectable naloxone adapted for IN delivery with a mucosal atomizer device. These IN naloxone delivery systems have been studied for reversing opioid-overdose [25]. The nasal mucosa is richly supplied with blood vessels and, in individuals who do not have underlying nasal disease or who have not recently used a vasoconstrictor, this may allow for rapid and adequate drug absorption. For example, in two randomized controlled clinical studies, IN naloxone was able to reverse OIRD in $74 \%$ and $72 \%$ of cases [25]. However, patients who received intramuscular (IM) naloxone responded more quickly than patients who received IN naloxone in terms of increased breaths per minute and two to four times as many IN reversals required supplementary naloxone compared to IM reversals [26, 27].

The distribution focus for IN naloxone has been heroin users, although patients taking opioids under medical supervision for chronic or cancer pain, as well as children in these households, actually comprise the larger population at risk for opioid emergencies [28]. Non-clinicians must be trained to administer naloxone properly as improper technique or dosing 
during an opioid emergency may result in incomplete opioid overdose reversal. Recently, a CDC report highlighted that additional models for distribution of naloxone to persons who might witness prescription opioid overdoses are needed [29]. With the available naloxone product limitations and the apparent need for additional naloxone distribution models, a novel naloxone auto-injector (EVZIO, kaleo, Inc., Richmond, VA) was designed and developed using human factors engineering (HFE) methods and guidelines. The auto-injector was developed as a "take-home" naloxone formulation that could be used outside of traditional healthcare settings by untrained individuals who are most likely to witness an opioid overdose emergency. The device is designed for only one injection, which provides a single therapeutic dose of $0.4 \mathrm{mg}$ naloxone (recommended range is $0.04 \mathrm{mg}$ to $2 \mathrm{mg}$ ) for reversing opioid overdose [17]. In addition, the single use design with audible prompts is a very important safety feature, which acts as an abuse deterrent and prevents repurposing the device with another syringe or injection device, prevents needle sharing use on another person, allows user to know exactly when the device has delivered a dose and when device is empty, and allows user to control dose and reduce likelihood of precipitating opioid withdrawal symptoms.

\section{Methods}

In the sphere of medical devices, HFE applies knowledge about human capabilities and limitations to the appropriate design and development of the device [30, 31]. HFE determines how and when a device would be used and how humans will interact with the device, seeking to minimize difficulties and pitfalls and optimize the likelihood of successful device use outcomes for the patient and end user. Thus, HFE may affect product design (e.g., buttons, covers, casings, alarms, labeling) to allow for optimal use and appropriate device function. This may include designing a product that allows for human limitations (such as poor eyesight, limited manual dexterity) as well as environmental conditions (e.g., lighting, stress, noise). The FDA recommends that HFE be applied early in the design process to allow for the most efficient, streamlined, and optimal product design possible, ensuring safe and effective use by mitigating or eliminating userelated hazards [31, 32]. HFE has been employed to optimize other similar devices for use by the general public, such as epinephrine auto-injectors [33].

Expanding access to naloxone for high-risk individuals and those around them is a key component of opioid harm reduction initiatives [34]. Any naloxone product must be designed to allow for rapid, accurate, and effective administration, thus human factors engineers played a critical role in the design of a novel take-home naloxone auto-injector intended for use by caregivers, family members, or other laypeople. The design of
EVZIO (naloxone hydrochloride injection) auto-injector was based on best practices in incorporating human factors analyses and rigorous verification and validation testing throughout the development process. Since this product is subject to use in an emergency situation by a diverse group of people with varying levels of skills and abilities, it was essential to create a product that is safe and easy to use, even for a person with no prior training or exposure to the product. Development activities were guided by the Quality System Regulation's Design Controls phases as contained in the Code of Federal Regulations, part 820.30 as well as FDA Guidance on the application Human Factors Engineering [31]. An iterative design philosophy was employed, encompassing the steps of research, design, risk assessment, review, and testing that then led to redesign, review, and further testing until the various potential hazards of the product could be mitigated to an acceptable level (Table 1).

The primary tasks associated with a successful injection using the naloxone auto-injector involve: 1) removing the outer case, 2) removing the safety guard, 3) placing the black (needle) end of the device on the outer thigh, 4) activating the device and holding it in place for at least $0.5 \mathrm{~s}$. Userelated hazards were evaluated during the development of this device by using several human factors methodologies including functional decomposition, formative usability evaluations, expert reviews, and heuristic analyses. Risks identified (e.g., user must understand quickly and accurately how to give the injection) during these steps shaped the final design of the auto-injector including device and outer case labeling.

\section{The auto-injector}

HFE and usability engineering processes were used to create a device that is portable (dimensions are approximately creditcard size with the thickness of a smartphone), ambidextrous in design, easy to handle, and use safely and effectively in almost any use environment during a suspected opioid emergency. The auto-injector's slim size allows it to be easily grasped by people with various hand sizes, including children, and allows for proper injection orientation. The outer case and device housing was built for durability with robust materials, providing impact resistance for the product. It contains a viewing window so that the user can readily confirm drug contents and use status (contents are clear prior to use and the window is obstructed following use). See Figs. 1 and 2. The needle in the naloxone auto-injector is shielded by a red safety guard that alerts the user to the location of the needle (red color implying a hazard) and provides instructions on how to remove the guard, which has tactile features to assure that it can be easily gripped and pulled off. The guard is designed in such a way that a degree of appropriate force is required to remove it, in order to ensure that the guard remains in place until the device is ready to be used. A sharps-injury prevention feature 
Table 1 The naloxone autoinjector's verification and validation test plan included testing associated and in compliance with FDA Guidance as well as the International Organization for Standardization (ISO): ISO 11608-1:2012, ISO 11608-2:2012, and ISO 116083:2012; other standard bodies such as UL, ASTM, and ISTA. In addition, tests were selected based on risk analyses. The following table provides a brief description of each test as well as its associated reference(s). This listing of tests is not a comprehensive list of all testing conducted for the naloxone autoinjector

\begin{tabular}{|c|c|}
\hline Description & References \\
\hline Dose Accuracy Test & ISO $11608-1$ \\
\hline \multirow[t]{2}{*}{ Environmental Exposure Test } & ISO $11608-1$ \\
\hline & Section 9.2.2 \\
\hline \multirow[t]{3}{*}{ Pre-Conditioning Test } & ISO 11608-1 \\
\hline & IEC 60068-2-30 \\
\hline & IEC 60068-2-6 \\
\hline \multirow[t]{3}{*}{ IEC Testing } & ISO 11608-1 (Section 10 and 11) \\
\hline & UL/IEC 60601-1-1 \\
\hline & IEC 60601-1-2 \\
\hline \multirow[t]{2}{*}{ Needle Bond Strength Test Method } & ISO 11608-2 (Section 11) \\
\hline & IEC 60068-2-30 \\
\hline Freedom From Leakage Test & ISO $11608-3$, Section 5.5. \\
\hline \multirow[t]{2}{*}{ Plunger Break/Glide Force Test } & ISO $11608-3$ \\
\hline & Section 5.6 \\
\hline Lock Out and Crush Test & FDA Draft Guidance on Injectors \\
\hline Injection Through Material Test & User FMEA \\
\hline Force to Remove Safety Guard & FDA Draft Guidance on Injectors \\
\hline Activation Force Test & DFMEA \\
\hline Battery Life Testing & User FMEA \\
\hline Sound Level Testing & User FMEA \\
\hline Device Accelerated Age Testing & $\begin{array}{l}\text { ISO } 11608 \text {, Medical Plastics and Biomaterials, July/August } 1998 . \\
\text { Pp. 16-23 }\end{array}$ \\
\hline $\begin{array}{l}\text { Safe Transit, Distribution, and Shipping } \\
\text { Testing }\end{array}$ & ISTA Guidelines ASTM D4169-08 \\
\hline Device Biocompatibility Testing & ISO 10993 \\
\hline Altitude Function Test & FDA Draft Guidance on Injectors \\
\hline Exposed Needle Length Test & ISO 11608 \\
\hline Label Wear Test & User FMEA \\
\hline Liquid Ingress Test & IEC 60529 \\
\hline Dispensing Time Test & DFMEA \\
\hline $\begin{array}{l}\text { Retraction Occurrence \& Visual } \\
\text { Inspection }\end{array}$ & ISO 11608-1 \\
\hline
\end{tabular}

user fmea: user failure mode and effects analysis, dfmea: design failure mode and effects analysis (retractable needle) was built into the device to assure the user never sees the needle before, during, or after naloxone administration thereby helping to also prevent inadvertent needle sticks post-injection. A naloxone formulation was specifically developed based upon the proven injectable formulation and tested, along with a new glass cartridge and elastomeric container closure system, to withstand temperature extremes up to 104 degrees for six months while maintaining naloxone stability.

Feedback from patients using opioids and their caregivers was utilized in order to optimize the labeling interface for the device, including the arrows, text placement, symbols, graphics, instructions found on the device, and on the Instructions for Use leaflet that accompanies the product. The largest possible font size and appropriate symbols were chosen to make the instructions as clear as possible without compromising regulatory labeling requirements. The autoinjector also offers redundancy in its instruction system by incorporating an innovative and smart electronic prompt system (the Intelliject Prompt System IPS®) that provides audible instructions for use from a speaker as well as visual cues in the form of LEDs in order to assist in guiding a user through the injection process. No special user activation is required for the audible prompts, which are automatically turned on and off by removing or replacing the outer case and advance with the user tasks at the user's pace, repeating if necessary. The prompts were carefully scripted to allow for quick, clear stepby-step actions in the case of an emergency and are delivered in a reassuring female voice. An acoustic chamber enhances the volume of the voice commands. The audible prompts tell the user what to do at each step and will repeat instructions if the user omits a critical step. When the injection is being 


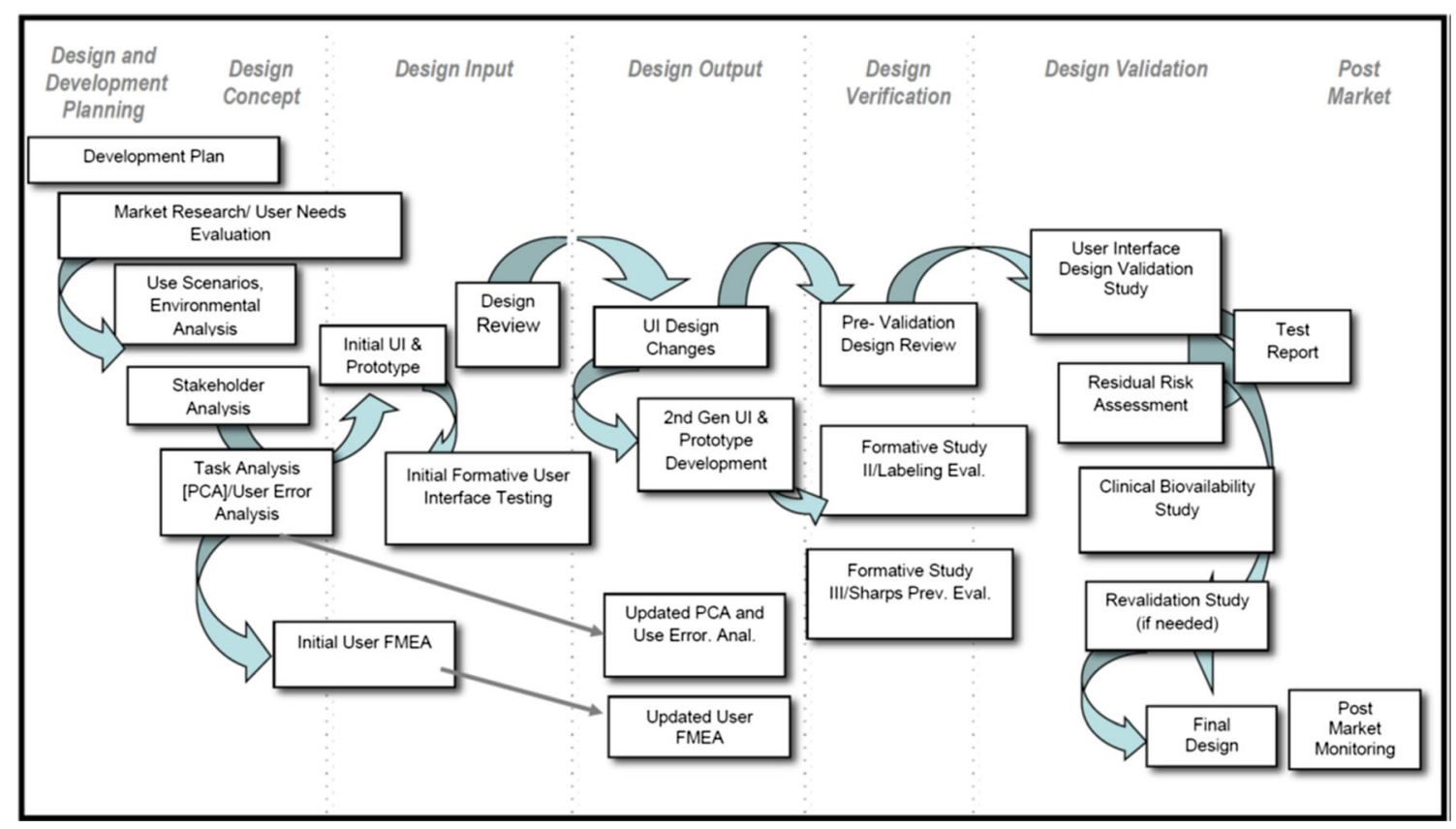

Fig. 1 EVZIO (naloxone hydrochloride injection) Auto-injector Human Factors Development Plan

administered, the device provides an audible countdown (e.g. $5,4,3,2,1)$ to help users hold the device in place to ensure complete naloxone delivery. When the injection is complete, the audible prompts confirm injection and remind the user to seek emergency medical attention. Post-use indicators tell the user that the device is used and should be properly discarded (Table 1).

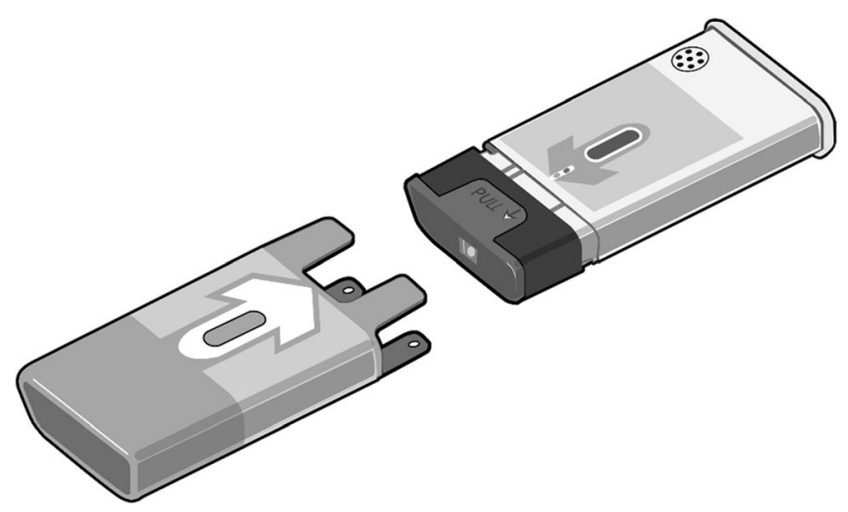

Fig. 2 The naloxone auto-injector is provided in a removable outer case. To increase performance success and decrease cognitive demand, the motion to slide off the outer case is in the same direction as the motion needed to pull off the safety guard. Once the outer case is removed, a removable red plastic safety guard shields the needle. A tactile design allows the safety guard to be gripped and pulled off when needed during an emergency. The outer case and the auto-injector have a viewing window to inspect the naloxone and to assess if the auto-injector has been used. The auto-injector also includes an electronic prompt system that provides voice instructions from a speaker and visual cues from LEDs to assist in guiding the user through the injection

\section{Training \& User Populations}

OIRD can develop gradually or suddenly into a lifethreatening emergency which may be fatal in a matter of minutes. During this situation an individual equipped with naloxone can use the product to quickly deliver a potentially lifesaving dose of parenteral naloxone. The naloxone autoinjector includes labeling on the device itself as well as an accompanying User Information leaflet. Moreover, a trainer device is included in each package so that a new user can practice the proper steps for using the auto-injector and train others on how to properly administer the product given that most patients are unresponsive during an opioid emergency requiring intervention from another individual such as a family member or other caregiver. The trainer for practice is similar in look and feel to the actual auto-injector device but does not contain a needle, the drug, or the activation mechanism for drug delivery. However, the trainer allows the user to practice the primary use tasks including removal of the outer case, the red safety guard, actuation of the black base, and become familiar with the audible prompts. In addition, the forces in the trainer were designed to mimic the forces used in the actual device in order to further facilitate correct use (e.g. safety guard removal force). The batteries in the trainer allow for repeated use once per day for several years if required. Of course, it is possible that situations could arise in which an individual with little or no training might have to use the auto-injector. For that reason the device and its user interface were specifically designed, validated, and approved to be used quickly by laypersons even without training. 
There are three main types of users of "take-home" naloxone. The first are caregivers to patients at increased risk for an opioid overdose emergency; these caregivers would be most likely to witness an opioid emergency in a home setting. While caregivers should be trained in the use of a naloxone product, it is nevertheless possible that an untrained family member might have to deliver the naloxone dose. A key concern in this scenario is that family members need to be able to locate the naloxone product quickly and use it correctly during an emergency. The next scenario involves a secondary caregiver, such as a coworker or friend. Ideally, secondary caregivers would receive training, but this may not always be the case; secondary caregivers may be more prone to getting flustered or overwhelmed by the emergency situation than primary caregivers. As a result, a product must be readily available and easy to understand, even if the user has not been trained recently or not trained at all. The third scenario involves a layperson who encounters a potential opioid overdose and is able to rapidly assess and respond to the emergency. If the layperson is a peer or drug user, he or she may have naloxone available or know to search for a naloxone product on the person. If the layperson is a random bystander, much depends on his or her ability to interpret the situation and search for the naloxone product. In the latter scenario, the layperson may fail to know about naloxone, and even if finding a product, may panic and not comprehend its use. However, for each of the above scenarios the naloxone auto-injector was specifically designed to be portable so that the product would be available on the patient or readily available for a caregiver in case of an opioid overdose emergency, and intuitive so that even if an untrained person finds the auto-injector, he or she would be able to use it properly and quickly. In addition, each naloxone auto-injector carton comes with two active devices so that multiple doses are available if required. The naloxone autoinjector can be used at most locations including home, work, restaurants, bars, outdoors, schools, on airplanes or other modes of transportation, at public gatherings such as concerts, and in places of entertainment. The naloxone auto-injector is not suitable for use in extreme environments, such as underwater or when exposed to extremely hot or cold temperatures for extended periods of time.

\section{Potential use errors and mitigation}

With any such product, especially in the case of products intended for administration during life-threatening emergencies, there is a hierarchy of use errors that range from critical (errors which could lead to a person not receiving a potentially life-saving dose of naloxone) to moderate (errors which could lead to a person possibly receiving an ineffective dose of naloxone or a significant delay in receiving naloxone) to minor (errors which might cause user frustration or a slight delay in administering naloxone). Conducting a thorough use risk assessment is an essential part of applying HFE to medical products and to obtaining FDA approval [31]. Potential use errors identified as a part of risk analyses conducted with the naloxone auto-injector have been summarized and categorized (Table 2).

As a part of developing the naloxone auto-injector using HFE, steps were taken to mitigate these aforementioned userelated risks. For example, the naloxone injection is administered in less than $0.5 \mathrm{~s}$, although product labeling and voice prompts require that the auto-injector be held in place for five seconds. The reason for this difference is that in emergency situations, users may panic and not hold the device in place long enough without a count-down that exceeds the injection time. The extra time the auto-injector is held in place does no harm and assures the dose is fully administered with complete needle retraction. As another example, the device and its outer case were designed with robust materials to be crush-resistant. This ensures that even if the product is dropped while being carried or the product is exposed to forces (e.g. sitting on the device) it will still function as intended.

The auto-injector specifies in labeling and in graphics that the injection is to be administered to the outer thigh and may be delivered through clothing. The outer thigh is an ideal injection site, but any number of other muscular locations (e.g., leg, upper arm, shoulder) would also be appropriate. This information is not provided on the device in an effort to streamline instructions and keep the use instructions simple during a highly stressful situation.

\section{Results: user-needs evaluations and randomized, controlled usability studies}

\section{Initial user-needs evaluation}

Nine adult caregivers (ranging in age from 32 to 66 years) were asked to use a prototype of the auto-injector device (without needle or drug) to inject an orange without any training as to how to use the product. As expected, use errors occurred on the prototype device as several users struggled to figure out how to remove the outer case or pull off the red safety guard. This feedback helped to refine final product design, labeling and the Instructions for Use. All (100\%) participants in this study believed the product was easy to use, and thought it was important to have, even if they did not think the patients they cared for were at high risk for opioid overdose. Additionally, all participants said the audible prompts were helpful.

\section{Labeling evaluation}

Seven current opioid patients and seven caregivers $(n=14)$ evaluated the auto-injector design and instructions. 
Table 2 Potential use errors for the naloxone auto-injector identified as a part of Human Factors Engineering and risk analyses

\begin{tabular}{|c|c|}
\hline Severity & Potential Use Errors \\
\hline \multirow[t]{7}{*}{ Minor } & User could misplace instructions, trainer device, or forget key steps \\
\hline & User drops device before or while removing the outer case \\
\hline & User does not understand how to open the package or has difficulty removing the packaging \\
\hline & User fails to hold the injector in place for the full five seconds \\
\hline & User ignores the instructional leaflet and uses the device anyway (untrained user) \\
\hline & User ignores the instructions on the device itself (untrained user) \\
\hline & User removes the safety guard prematurely and then tries to replace it \\
\hline \multirow[t]{7}{*}{ Moderate } & User moves device during the injection, leading to possibility of ineffective dose \\
\hline & User misplaces the device or cannot find it readily \\
\hline & User has difficulty pulling off the safety guard \\
\hline & $\begin{array}{l}\text { User does not apply sufficient force initially to activate the injection then eventually determines } \\
\text { force required }\end{array}$ \\
\hline & User has difficulty removing the device from the outer case \\
\hline & $\begin{array}{l}\text { User attempts to disassemble the device, causing the possibility of premature activation which } \\
\text { might result in unintentional self-injection }\end{array}$ \\
\hline & $\begin{array}{l}\text { User removes the safety guard and then drops the device, causing the potential for premature } \\
\text { activation }\end{array}$ \\
\hline \multirow[t]{11}{*}{ Critical } & User employs the device but the drug is expired or degraded \\
\hline & User attempts to use a previously used device \\
\hline & User does not remove the red safety guard \\
\hline & $\begin{array}{l}\text { User pulls the device away from the outer thigh prematurely or fails to hold the injector in place for } \\
\text { at least } 0.5 \mathrm{~s}\end{array}$ \\
\hline & User decides not to give the injection \\
\hline & User places the device in the wrong location (not on the outer thigh or an appropriate muscle) \\
\hline & User does not place the base on the outer thigh or appropriate alternate location \\
\hline & User never applies sufficient force to activate the injection \\
\hline & User puts hand over the wrong end, possibly resulting in accidental self-injection \\
\hline & User pushes the base with fingers causing premature activation \\
\hline & User uses the training device on the patient instead of the actual device containing dru \\
\hline
\end{tabular}

Participants were provided various labeling designs and given randomized levels of training (ranging from no training at all to full training including review of the Instructions for Use leaflet). Subjects ranged in age from 30 to 65 years. All 14 participants were asked to simulate an injection into a mannequin; $100 \%$ could do so successfully. It should be noted that $2 / 14$ participants had a close call in completing the tasks correctly but adjusted their actions based on voice prompts and completed the injection properly. All participants rated use as "completely easy," "easy," or "slightly easy." No participant reported the device was "difficult" to use in any way.

\section{User Interface design validation study}

Forty untrained adult (age $\geq 20$ years) and juvenile (ages 12 to 19 years) participants were asked to take the naloxone auto-injector without being exposed to the product or any associated instructional leaflets/training and administer an injection into a mannequin. Participants were read the scenario and told that the study was designed to simulate a "real-life opioid emergency." Ninety percent of participants were able to do so properly. Of the $10 \%$ of participants who failed to deliver the injection without any training, the use errors were failure to press the device firmly enough to engage the injection mechanism $(n=2)$, use of the trainer device instead of the actual device $(n=1)$, and failure to hold the device in place for one full second $(n=1)$.

The average time to complete the simulated injection from selection of the device to completed injection was $64.0 \mathrm{~s}$ for adults and $57.6 \mathrm{~s}$ for juveniles. When asked to rate the difficulty of administering the injection, $70 \%$ rated every step in the process as "easy" or "very easy." Twenty percent rated only one of the steps as "hard" and the rest as "easy" or "very easy." Of the juvenile subjects, $10 \%$ rated more than one of the steps as "hard." One area that challenged participants was difficulty removing the safety guard, leading to some of the lower ratings. However, one participant stated that the red 
safety guard should be somewhat difficult to remove since it is a safety feature.

When asked if they would be confident using the device again, all of the test participants (100\%) said they would. Importantly, all participants stated that they would call for emergency help either before or after using the auto-injector. Participants were asked for their opinion about the voice prompts. The majority ( $83 \%$ ) rated voice prompts as a positive feature and more than half $(62.5 \%)$ rated it as the first of the device's positive features. In a post-test risk assessment for critical use errors, that is, errors that in a real-world situation might have led to failure to administer naloxone properly, $90 \%$ of participants would have been able to administer the naloxone appropriately without training.

\section{Comparative usability studies}

Two randomized, controlled head-to-head usability studies (one located in Pennsylvania and another in Massachusetts) comparing the naloxone auto-injector to an improvised intranasal (IN) naloxone delivery system were conducted to assess correct administration of naloxone during a simulated opioid emergency. The studies assessed participants' ability to successfully administer naloxone without training or exposure to the product and associated instructional materials as well as whether participants retained the ability to correctly administer naloxone a week or more following training. The simulation included a home environment scenario with distractions added to induce stress to the environment and encompassed three phases. In Phase I, each study participant was read a scenario by the moderator reflecting a common opioid overdose emergency situation. Participants then needed to administer naloxone to a mannequin (randomly assigned face-up or face-down) using the product found on a table in accordance with its labeling with no other training or instructional materials provided. This approach was used to model a real life scenario where a family member or caregiver of an opioid overdose victim may need to administer naloxone without any formal training or prior exposure to the naloxone delivery system. Participants were randomly assigned to either naloxone auto-injector or the IN naloxone delivery system first and the other naloxone product second to complete Phase I. Use of a naloxone product was considered complete when the study participant indicated that he/she had finished or upon $15 \mathrm{~min}$ having elapsed during the participant's attempt to use the naloxone product. Phase II occurred immediately after Phase I and involved training of each study participant on proper use of the naloxone auto-injector and the IN naloxone delivery system by a trained healthcare professional. Participants were required to demonstrate correct use of both naloxone products for Phase II completion. Study participants returned no sooner than seven days later for Phase III. In Phase III, each study participant repeated the simulated opioid overdose emergency scenario from Phase I. Participants were required to recall the training that was given during Phase II as no additional training or instructional materials were provided before or during Phase III except for labeling on the naloxone products.

Successful administration was defined as whether the study participant would have administered the intended dose of naloxone. In phase 1, $94 \%$ of participants could complete a successful injection using the naloxone auto-injector versus $0.0 \%$ of those using the IN naloxone delivery system $(p<0.0001)$. In phase III, $100 \%$ of participants completed a successful injection using the naloxone auto-injector versus $50.6 \%$ using the IN naloxone delivery system $(\mathrm{p}<0.0001)$. The average time for successful naloxone administration in Phase I with the naloxone auto-injector was $56 \mathrm{~s}$ and an average time was not available for the IN naloxone delivery system as no participants successfully administered the IN naloxone [35]. After excluding Phase III completion times for study participants who were unsuccessful in administering naloxone for any reason, study participants were able to successfully administer naloxone 3 times faster with the naloxone autoinjector (35 s) as compared to the IN naloxone system (88 s).

\section{Discussion}

Opioid emergencies, including overdose, are frequently witnessed and many occur at home [36]. To our knowledge, the concept of providing naloxone for the out-of-hospital administration for opioid overdose emergencies was first proposed in the literature in 1996 [37]. While much emphasis has been placed on reducing overdose mortality among the injection drug-using population and those using opioids nonmedically, the population of patients prescribed opioid analgesics for control of chronic or cancer pain, including their children, comprises the larger group at increased risk for an opioid emergency [28]. These patients may benefit the most from the novel auto-injector in that they are the more numerous group and likely live with family and caregivers who can be equipped with the auto-injector and trained in its proper use. Prior emergency naloxone delivery models relied on IN or IM dosing and required specific assembly or delivery steps that were not always intuitive to inexperienced users. These naloxone kits worked well with community groups who were able to train volunteers to introduce the kits into the street drug community but have limitations for the broader layperson community.

Until the naloxone auto-injector, there was no FDAapproved product that could be readily available and used in the out-of-hospital setting by laypersons. Thus, this novel naloxone product meets a large need in a particularly userfriendly way. Analogous to an automated external defibrillator, the naloxone auto-injector is designed to be used by virtually anyone: trained or untrained caregivers, family 
members, friends, bystanders, and others suddenly confronted with a life-threatening opioid overdose emergency; and was developed using a robust HFE process so that it is can be used virtually anywhere: at home, in the office, while shopping or traveling, indoors or outdoors. The auto-injector was carefully designed to help mitigate situational aspects (e.g., use environments, noise level) that could exacerbate the considerable stress that could be experienced by the caregiver or bystander during an opioid emergency. For that reason, testing of the auto-injector was conducted under a variety of use conditions (e.g., home use, distractions) and under a variety of simulated weather conditions (e.g., cold weather, rain, heat, high humidity). In all normal or even extreme conditions (e.g., between the temperature range $4{ }^{\circ} \mathrm{C}$ and $40^{\circ} \mathrm{C}$ ), the device was verified to function properly. This degree of versatility is an important aspect to the utility of the auto-injector.

Prescribers must recognize that life-threatening opioid emergencies characterized by OIRD are not rare events. They affect patients as well as heroin users taking prescribed opioid pain relievers under medical supervision. These emergencies may also affect small children through accidental exposure. In the event of an opioid overdose emergency, the ability of a person on the scene, regardless of age, training, or medical background, to administer an effective dose of naloxone through the proven injectable route of administration, combined with an easy to use delivery system, has the clear potential to save lives. As such, the naloxone autoinjector could be a universal precaution for those who prescribe opioid therapy. Family members or caregivers of the opioid patient can be trained on the simple steps to deliver a naloxone injection by using the Trainer for practice as suggested in the product approved labeling. Even without training, studies have demonstrated that most people can correctly administer naloxone using the auto-injector.

\section{Conclusion}

The increased use of opioids for chronic treatment of pain and the resulting epidemic of opioid overdoses have created a major public health challenge. Parenteral naloxone has been used for over 40 years to treat opioid overdose. In 2014, FDA approved a novel naloxone auto-injector device (EVZIO, kaleo, Inc., Richmond, VA) that can be prescribed for "take-home" use and administered parenterally by laypeople. Since naloxone must be administered quickly, under highly stressful conditions, and possibly by inexperienced laypeople, it was crucial to design and develop a product that incorporated HFE, risk analyses, and actual testing of user-device interaction without training. Developing a drug-device combination product requires a rigorous development process that, in the case of the naloxone autoinjector, involved more than 100 engineering verification tests, multiple usability methodologies and studies. Applying a robust development process incorporating HFE resulted in a product that was designed to be safe, fast, easy and predictably reliable to deliver a potentially life-saving dose of naloxone during an opioid overdose emergency.

Acknowledgments This paper was prepared with editorial support from Mark Elzey, MD, who is employed by kaleo, Inc., Richmond, Virginia as well as LeQ Medical, Angleton, Texas, whose services were paid for by the authors.

\section{Compliance with ethical standards}

Conflict of interest disclosure This paper was prepared by NEMA Research Inc. medical writing staff. NEMA Research Inc. received funding from kaleo, Inc. for the preparation of this manuscript. Dr. Nalamachu and Dr. Pergolizzi are consultants for kaleo Inc. Dr. Raffa is a consultant for NEMA Research. Dr. Taylor is an employee of NEMA Research. Dr. Elzey and Dr. Edwards are employees of kaleo Inc.

\section{References}

1. Daher M. Pain relief is a human right. Asian Pacific J Cancer Prev : APJCP. 2010;11(Suppl 1):97-101.

2. Volkow ND. Prescription Opioid and Heroin Abuse. National Institute on Drug Abuse. https://www.drugabuse.gov/aboutnida/legislative-activities/testimony-to-congress/2015/prescriptionopioid-heroin-abuse. Accessed 3 Mar 2016. 2014.

3. Centers for Disease Control and Prevention. Trends in DrugPoisoning Deaths Involving Opioid Analgesics and Heroin: United States, 1999 to 2012. In: NCHS Health E-Stat. Centers for Disease Control and Prevention,, Atlanta, GA. http://www.cdc. gov/nchs/data/hestat/drug_poisoning/drug_poisoning.htm. Accessed 27 Jul 2015. 2014.

4. Centers for Disease Control and Prevention. QuickStats: Rates of Deaths from Drug Poisoning and Drug Poisoning Involving Opioid Analgesics-United States, 1999-2013. Morbidity and Mortality Weekly Report. 2015;64(1):32.

5. Warner M, Chen L, Makuc D, Anderson R, Minino A. Drug poisoning deaths in the United States, 1980-2008. Hyattsville, MD: National Center for Health Statistics 2011.

6. Manchikanti L, Helm 2nd S, Fellows B, Janata JW, Pampati V, Grider JS, et al. Opioid epidemic in the United States. Pain Phys. 2012;15(3 Suppl):ES9-38.

7. Hall AJ, Logan JE, Toblin RL, Kaplan JA, Kraner JC, Bixler D, et al. Patterns of abuse among unintentional pharmaceutical overdose fatalities. JAMA : J Am Med Assoc. 2008;300(22):2613-20. doi:10.1001/jama.2008.802.

8. Paulozzi L. CDC: grand rounds: prescription drug overdosesA U.S. epidemic. Morb Mortal Wkly Rep. 2012;61(01):10-3.

9. Zedler B, Xie L, Wang L, Joyce A, Vick C, Kariburyo F, et al. Risk factors for serious prescription opioid-related toxicity or overdose among veterans health administration patients. Pain Med. 2014;15(11):1911-29.

10. Paulozzi LJ. Prescription drug overdoses: a review. J Saf Res. 2012;43(4):283-9. doi:10.1016/j.jsr.2012.08.009.

11. Yokell M, Delgardo M, Zaller N, Wang N, McGowan S, Green T. Presentation of prescription and nonprescription opioid overdoes to US emergency departments. JAMA Internal Med. 2014;174(12): 2034-7. 
12. Burghardt LC, Ayers JW, Brownstein JS, Bronstein AC, Ewald $\mathrm{MB}$, Bourgeois FT. Adult prescription drug use and pediatric medication exposures and poisonings. Pediatrics. 2013;132(1):18-27. doi:10.1542/peds.2012-2978.

13. Alliance FC. Hypoxic-Anoxic Brain Injury. 2004. https://www. caregiver.org/hypoxic-anoxic-brain-injury. Accessed 3 Mar 2016.

14. Agamanolis DP. Cerebral Ischemia and Stroke. In: Neuropathology: An Illustrated Interactive Course for Medical Students and Residents. 2013. http://neuropathology-web. org/chapter2/chapter2aHIE.html. Accessed 6 Mar 2016.

15. The National Emergency Medical Services for Children Data Analysis Resource Center. National EMS Information System (NEMSIS Data): Average EMS Response Times, Scene Times, and Transport Times. 2012. http://www.nedarc.org/emsDataSystems/nemsisReports/2010_11 EMSTimes.html. Accessed 3 Mar 2016.

16. Green TC, Heimer R, Grau LE. Distinguishing signs of opioid overdose and indication for naloxone: an evaluation of six overdose training and naloxone distribution programs in the United States. Addiction (Abingdon, England). 2008;103(6):979-89. doi:10.1111 jj.1360-0443.2008.02182.x.

17. Kim HK, Nelson LS. Reducing the harm of opioid overdose with the safe use of naloxone : a pharmacologic review. Expert Opin Drug Saf. 2015;14(7):1137-46. doi:10.1517/14740338.2015.1037274.

18. Mueller SR, Walley AY, Calcaterra SL, Glanz JM, Binswanger IA. A review of opioid overdose prevention and naloxone prescribing: implications for translating community programming into clinical practice. Substance Abuse. 2015:1-14. doi:10.1080/08897077.2015.1010032.

19. Community Management of Opioid Overdose. Geneva: World Health Organization. 2014.

20. Strang J, McDonald R, Tas B, Day E. Clinical provision of improvised nasal naloxone without experimental testing and without regulatory approval: imaginative shortcut or dangerous bypass of essential safety procedures? Addiction (Abingdon, England). 2016;111(4):574-82. doi:10.1111/add.13209.

21. Rowe C, Santos GM, Vittinghoff E, Wheeler E, Davidson P, Coffin PO. Predictors of participant engagement and naloxone utilization in a community-based naloxone distribution program. Addiction (Abingdon, England). 2015. doi:10.1111/add.12961.

22. Walley AY, Xuan Z, Hackman HH, Quinn E, Doe-Simkins M, Sorensen-Alawad A, et al. Opioid overdose rates and implementation of overdose education and nasal naloxone distribution in Massachusetts: interrupted time series analysis. BMJ (Clin Res). 2013;346:f174. doi:10.1136/bmj.f174.

23. Project Lazarus. Community-based overdose prevention and opioid safety with community care of North Carolina. Project Lazarus, Moravian Falls, NC. 2015. http://www.projectlazarus.org//. Accessed 27 Jul 2015.

24. Bennett AS, Bell A, Tomedi L, Hulsey EG, Kral AH. Characteristics of an overdose prevention, response, and naloxone distribution program in Pittsburgh and Allegheny County,
Pennsylvania. J Urban Health : Bull N Y Acad Med. 2011;88(6): 1020-30. doi:10.1007/s11524-011-9600-7.

25. Robinson A, Wermeling DP. Intranasal naloxone administration for treatment of opioid overdose. Am J Health-Syst Pharm : AJHP : Off J Am Soc Health-Syst Pharm. 2014;71(24):2129-35. doi:10.2146 /ajhp130798.

26. Kerr DR, Kelly A-M, Dietze P, Jolley D, Barger B. Randomized controlled trial comparing the effectiveness and safety of intranasal and intramuscular naloxone for the treatment of suspected heroin overdose. Addiction (Abingdon, England). 2009;104(12):2067-74.

27. Kelly AM, Kerr DR, Dietze P, Patrick I, Walker T, Koutsogiannis Z. Randomised trial of intranasal versus intramuscular naloxone in prehospital treatment for suspected opioid overdose. Med J Aust. 2005;181(1):24-7.

28. Centers for Disease C, Prevention. CDC grand rounds: prescription drug overdoses - a U.S. epidemic. MMWR Morb Mortal Wkly Rep. 2012;61(1):10-3

29. Wheeler E, Jones TS, Gilbert MK, Davidson PJ. Opioid overdose prevention programs providing naloxone to laypersons - United States, 2014. MMWR Morb Mortal Wkly Rep. 2015;64(23):631-5.

30. FDA. Medical Devices; Current Good Manufacturing Practice (CGMP) Final Rule; Quality System Regulation. Food and Drug Administration, Rockville, MD. 1996. http://www.fda. gov/medicaldevices/deviceregulationandguidance/postmarket requirements/qualitysystemsregulations/ucm230127.htm. Accessed 31 May 2015

31. Food and Drug Administration. Applying Human Factors and Usability Engineering to Medical Devices: Guidance for Industry and Food and Drug Administration Staff In: Center for Devices and Radiological Health, editor. 2016.

32. Story M. FDA Human Factors Draft Guidance Document: Agency Expectations for Human Factors Data in Premarket Submission. Rockville, MD: US Food and Drug Administration; 2012. p. 22 May.

33. Edwards E, Edwards E, Simons F, North R. Drug-device combination products in the twenty-first century: epinephrine auto-injector development using human factors engineering. Expert Opin Drug Deliv. 2015;12(5):751-62.

34. Wermeling DP. Review of naloxone safety for opioid overdose: practical considerations for new technology and expanded public access. Ther Adv Drug Saf. 2015;6(1):20-31. doi:10.1177 12042098614564776.

35. Edwards ET, Edwards ES, Davis E, Mulcare M, Wiklund M, Kelley G. Comparative usability study of a novel auto-injector and an intranasal system for naloxone delivery. Pain Ther. 2015. doi:10.1007/s40122-015-0035-9.

36. Sporer KA. Acute heroin overdose. Ann Intern Med. 1999;130(7): 584-90.

37. Strang J, Darke S, Hall W, Farrell M, Alt R. Heroin overdose: the caes for take-home naloxone. BMJ (Clin Res). 1996;312:1435-6. 